Superalloys 2012: $12^{\text {th }}$ International Symposium on Superalloys

\title{
DEFORMATION MECHANISMS COUPLED WITH PHASE FIELD AND CRYSTAL PLASTICITY MODELING IN A HIGH TEMPERATURE POLYCRYSTALLINE NI-BASED SUPERALLOY
}

\author{
H.Z. Deutchman ${ }^{1}$, P.J. Phillips ${ }^{1,2}$, N. Zhou ${ }^{1,3}$, M.K. Samal ${ }^{4}$, S. Ghosh ${ }^{4}$, Y. Wang ${ }^{1}$ and M.J. Mills ${ }^{1}$ \\ ${ }^{1}$ The Ohio State University, Department of Materials Science and Engineering, Columbus, OH 43210, USA \\ ${ }^{2}$ Now at the University of Illinois at Chicago, Department of Physics, Chicago, IL 60608, USA \\ ${ }^{3}$ Now at GE Global Research Center, Niskayuna, NY 12309, USA \\ ${ }^{4}$ Johns Hopkins University, Department of Civil Engineering, Baltimore, MD 21218, USA
}

Keywords: Ni-based Superalloy, Creep, STEM, Deformation Substructure, Crystal Plasticity, Phase Field

\begin{abstract}
Physics-based modeling of disk superalloys is inherently complex due to the strong influence of microstructure on properties, as well as the multitude of deformation mechanisms operative at elevated temperatures. The present contribution will focus on the effects of monotonic and cyclic loading conditions, and the underlying deformation mechanisms will be discussed. Detailed substructure analysis of deformed specimens was conducted with scanning transmission electron microscopy diffraction-contrast methods. These characterization efforts have led to a series of phase field simulations in which the interaction of various deformation modes with experimentally measured precipitate configurations can be explored and critical parameters quantified. Additionally, these results have been incorporated into a novel, dislocation-densitybased crystal plasticity model that has been calibrated based on a single crystal response, and enables the computationally efficient modeling of polycrystalline behavior.
\end{abstract}

\section{Introduction}

As engine operating temperatures climb higher with the aim of better fuel efficiency, loading behavior and disk alloys become more complex. With this increased complexity, the ability to accurately model behavioral output with alloy input will become an invaluable tool for the aviation industry. The newest generation of alloy behavioral modeling must be based on not only accurately recreating the mechanical behavior curves, but also on an understanding of the effects of alloy parameters on deformation mechanisms. Polycrystalline Ni-based superalloys are the workhorse for turbine disk alloys due to their increased resistance to creep and fatigue at intermediate temperatures. The $\gamma / \gamma^{\prime}$ microstructure provides a rich environment for many different deformation mechanisms to thrive. This paper highlights microstructural characterization, mechanical behavior, substructure analysis, and modeling efforts to make connections between the observations, in the hopes of progressing towards a general model of polycrystalline disk behavior.

\section{Experimental Procedure}

All creep and constant strain rate samples were provided by GE Aviation, in the form of smooth bars with an average $26 \mathrm{~mm}$ gage length and $4 \mathrm{~mm}$ diameter. The polycrystalline disk alloy ME3 was used for the tests, after a heat treatment that produced a bimodal $\gamma$ ' microstructure, shown in Figure 1. The average grain size provided was ASTM $7(30 \mu \mathrm{m})[1]$. Prior to any testing, a full examination of the microstructure was completed. Samples were mechanically polished progressively with a final colloidal silica vibratory polishing step for several days. The samples were then briefly etched to remove $\gamma$, particles, using a solution of hydrofluoric acid, nitric acid, and lactic acid. Images were captured using the back-scattered electron detector in combination with the ultra-high resolution feature on an FEI Sirion SEM. The SEM images were then analyzed using the Fovea Pro plug-in for Adobe Photoshop. A full explanation of these procedures can be found in Payton et al. [2].

The supersolvus heat treatment, followed by cooling and standard aging, produced a combined secondary and tertiary $\gamma^{\prime}$ volume fraction of approximately $50 \%$. The average size of the secondary $\gamma^{\prime}$ was $223 \mathrm{~nm}$ (45\% volume fraction), and the average tertiary $\gamma^{\prime}$ size was $22.6 \mathrm{~nm}$ ( $5 \%$ volume fraction).

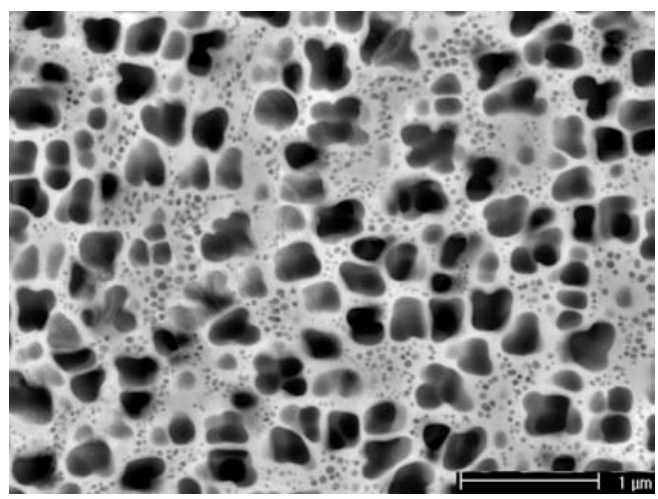

Figure 1. Bi-modal ME3 microstructure.

Monotonic creep tests were performed at $704{ }^{\circ} \mathrm{C}$ and a range of stresses from 655-793 $\mathrm{MPa}$, with increments of $34 \mathrm{MPa}$. All creep tests were completed to approximately $0.5 \%$ creep strain with the aim of performing post-creep substructure analysis. Linear variable displacement transducers (LVDT) were used to measure a change in voltage, which was then converted to a creep strain value. Temperature was measured by two thermocouples attached to the LVDT cage, placed directly on either side of the sample. The tests were heated under preload to prevent any slack in the loading arm, and then cooled under load to preserve the deformation microstructure.

Constant strain rate testing was performed on an MTS 810 tensile frame. All tests were performed at $704{ }^{\circ} \mathrm{C}$, and $10^{-4} \mathrm{~s}^{-1}$ strain rate. The goal of these experiments was to compare the deformation mechanisms observed under creep conditions to those observed under more rapid constant strain rate conditions. All tests were terminated at small strain levels in order to enable more direct comparison between testing modes. After the test reached the desired displacement, the sample was cooled rapidly (by opening the clam-shell furnace) while under load control in order to preserve the deformation substructure. 


\section{Characterization Methods}

After testing, each polycrystalline sample was cut at a $45^{\circ}$ angle from the tensile axis in order to view deformation along the slip systems that experience the highest shear stresses. The slices were then polished down to approximately $100 \mu \mathrm{m}$ and slurry drilled to produce $3 \mathrm{~mm}$ TEM disks. Finally, the samples were electropolished with a solution containing methanol, butoxyethanol, and perchloric acid at $20 \mathrm{~V}$ and $-30{ }^{\circ} \mathrm{C}$.

All thin foils were then examined via scanning transmission electron microscopy (STEM) on an FEI Tecnai F20 S/TEM operated at $200 \mathrm{kV}$. The use of STEM in diffraction contrast analysis has previously been presented as a valid means of defect characterization, and has demonstrated advantages over conventional TEM [3]. Such benefits include the ability to analyze thicker samples, and to image defects while in a zone axis orientation. The latter allows for imaging multiple defect systems within a given grain simultaneously as multiple diffraction vectors are excited. All of the following images were acquired in either a systematic row or zone axis diffraction condition.

\section{Mechanical Behavior and Substructure Studies}

\section{Creep Test Results}

Figure 2 below shows the creep strain percent versus time (hours) of each creep test. Aside from the highest stress (793 MPa), each creep curve bottoms out with a brief negative region, exhibiting a minimum strain rate. Following this region, the strain rate increases significantly. In traditional terms, the polycrystalline Ni-based superalloy does not exhibit traditional "secondary creep," and instead goes right to "tertiary creep." At the small strains examined, the strain rate increased with strain. Only the $793 \mathrm{MPa}$ test exhibited an increasing strain rate with strain.

In post-deformation STEM analysis it appeared that there were two major categories of deformation. The lower stress tests $(655$ MPa-724 MPa) displayed a dominance of reorder-mediated deformation, especially microtwins. This has been observed in similar alloys and testing conditions [4]. Higher stress tests (758 MPa-793 MPa) showed non-planar and planar activity.

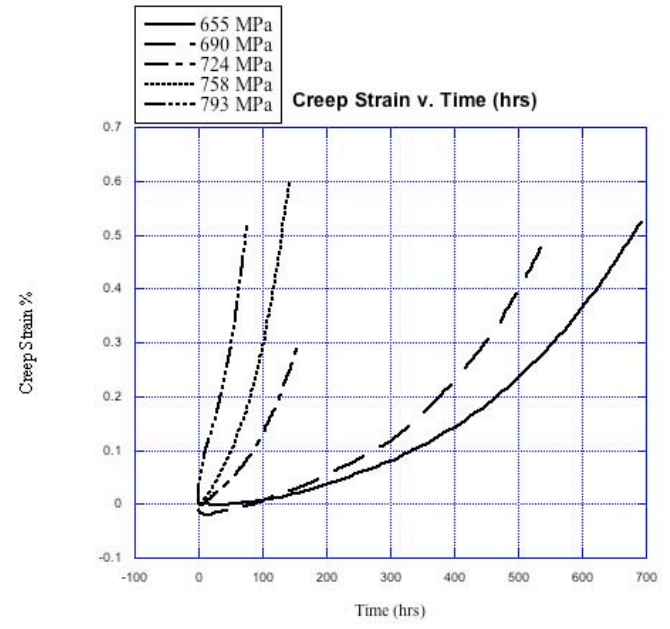

Figure 2. Creep strain percent versus time (hours).
Deformation Mechanism Characterization - Low Stress At the lowest stresses (655 MPa-724 MPa), deformation was dominated mostly by microtwinning, stacking faults in the $\gamma^{\prime}$, and paired and unpaired dislocation activity. Twinning and isolated stacking faults can be attributed to reorder-mediated processes, and reorder-mediated faulting can occur for paired shearing at elevated temperatures. Microtwins are thin structures that are continuous through the grains. Kovarik et al [5] postulated that when matrix dislocations dissociate into Shockley partials, shearing of the $\gamma$ ' particles by identical $a / 6<112>$ partials on adjacent $\{111\}$ planes would create a high-energy fault with two complex stacking faults. In order to enable this shearing mode to continue, a reordering process must occur to remove the resulting chemical fault. The local diffusional process associated with reordering leads to the creation of a two layer extrinsic stacking fault that is left over from the structural part of the complex extrinsic stacking fault, and repetition of this process on adjacent $\{111\}$ planes leads to the creation of the thin mechanical twins. Unocic et al [6] also determined that decorrelation of $a / 2<110\rangle$ dislocations is a necessary precursor to microtwinning. Figure 3 presents microtwins in both lower stress creep tests.

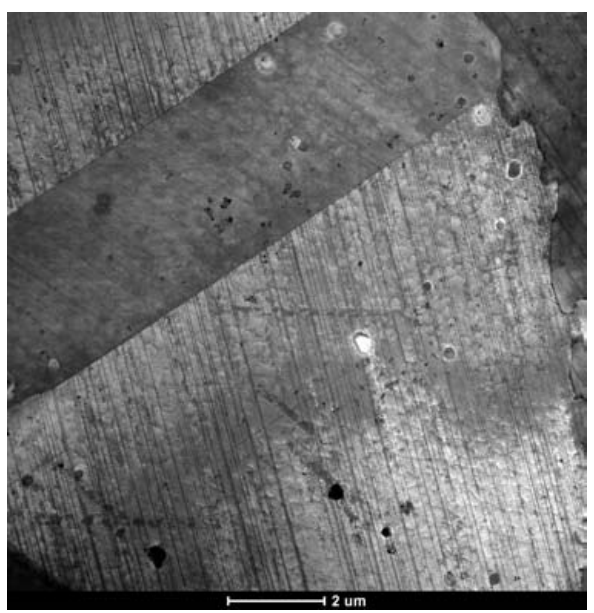

(a)

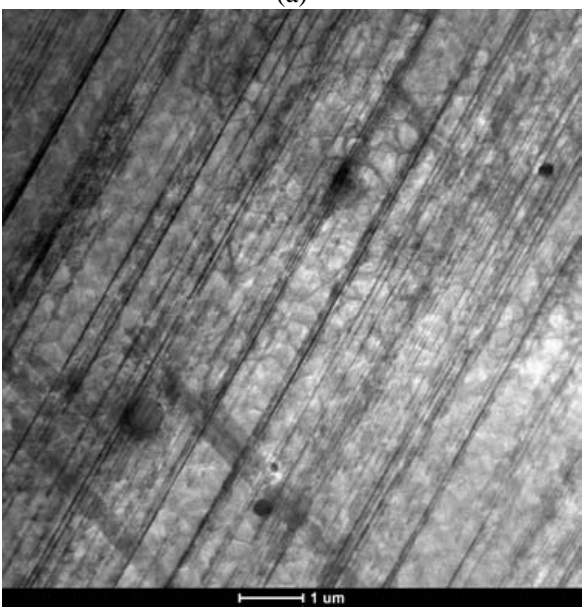

(b)

Figure 3. STEM imaging of a) microtwins in $655 \mathrm{MPa}$ sample and b) microtwins in $724 \mathrm{MPa}$ sample. 
It is thought that isolated faults are formed when two $a / 2<110\rangle$ matrix dislocations with different Burgers vectors travel on two adjacent $\{111\}$ planes. They are able to shear the $\gamma$ ' and reorder to correct wrong nearest neighbors. In doing so, they leave behind a stacking fault and an $a / 6<112>$ loop around the $\gamma^{\prime}$ precipitate, and the two $a / 2<110\rangle$ dislocations are able to keep moving [6]. Figure 4 a below shows isolated faulting in a lower stress creep test.

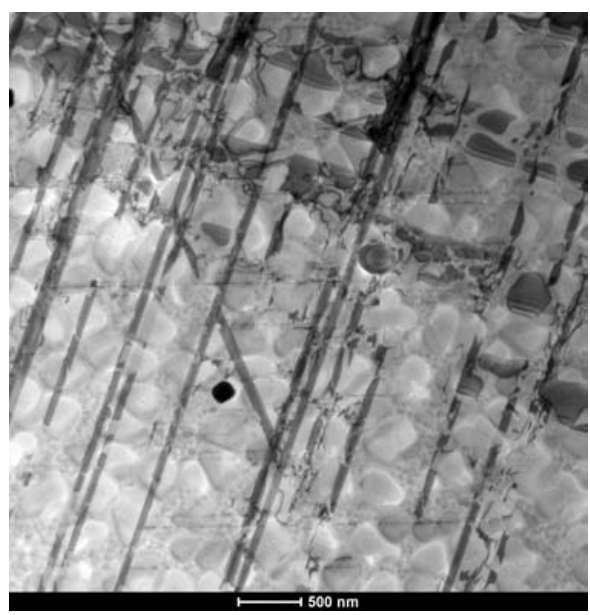

(a)

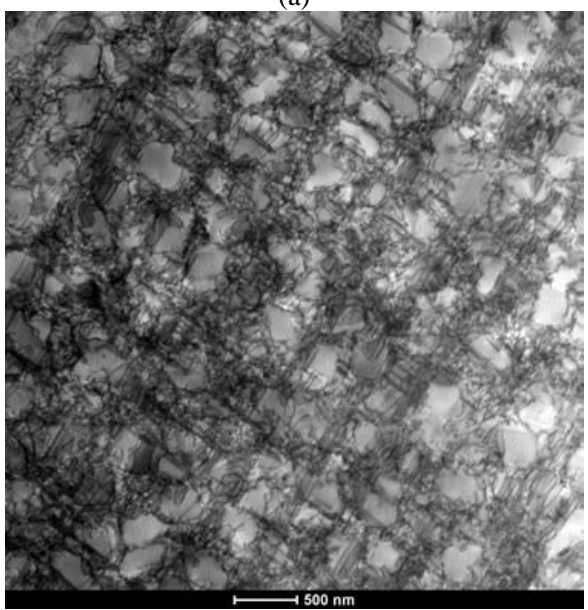

(b)

Figure 4. STEM imaging of a) isolated faulting b) planar dislocation bands passing through matrix and precipitate regions in $655 \mathrm{MPa}$ creep sample.

With enough temperature and time, unpaired dislocations can climb over the $\gamma$ ' precipitates. The diffuse dislocation bands shown in Figure $4 \mathrm{~b}$ are caused by local climb, which allows for the traveling dislocations to move to different, parallel slip planes. In the lower stress samples, these bands are parallel, as shown below in Figure 4b. A homogenous distribution of coupled dislocations in the $\gamma$ matrix can be attributed to paired shearing.

Deformation Mechanism Characterization - High Stress As the creep stress was increased to $758 \mathrm{MPa}-793 \mathrm{MPa}$, there was a significant deformation mechanism change. Microtwins still appeared as an important mechanism (Figure 5a), but non-planar, "wavy" slip was also observed at these higher stresses (Figure $5 b)$. Wavy slip will be discussed in more detail with respect to the constant strain rate tests in the following section. This mechanism is also active in fatigue samples of ME3 with the same heat treatment-produced microstructure, and at similar temperatures [7]. Paired dislocations are also associated with wavy slip. The paired dislocation activity suggests only APB shearing exists at the higher stresses.

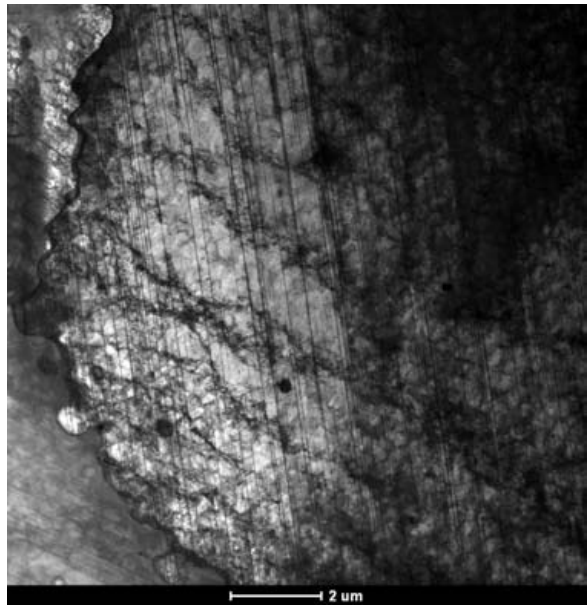

Figure 5. STEM imaging of planar activity and wavy slip in 793 $\mathrm{MPa}$ creep sample.

\section{Constant Strain Rate Testing}

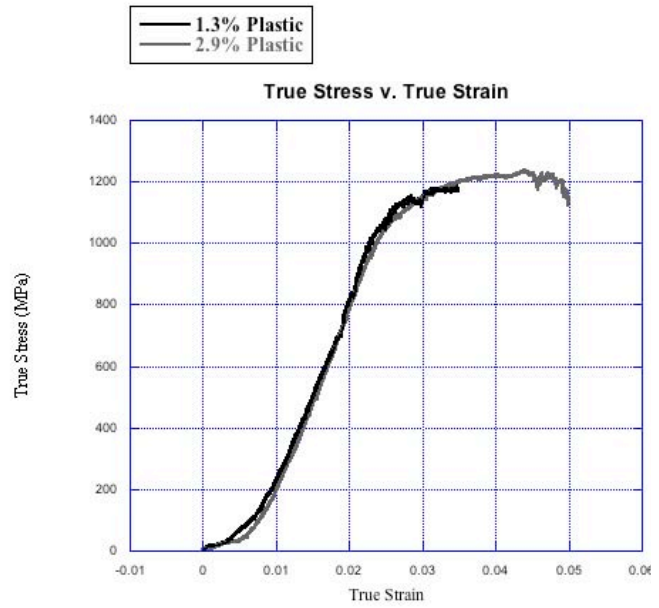

Figure 6. True Stress v. True Strain, constant strain rate tests.

Constant strain rate samples deformed to approximately $1.3 \%$ and $2.9 \%$ plastic strain show similar deformation mechanisms. In contrast to the creep samples, however, there is intense dislocation activity present following constant strain rate testing. The images in Figure 7 provide a representative overview of the various types of deformation observed. One of the most active mechanisms that appear in the microstructure is dislocation activity. The degree of 
coupling changes, showing some dislocations that appear strongly paired, whereas in other cases they appear to be rather weakly coupled. Increased dislocation content was observed at $2.9 \%$, which is expected given the increased plastic strain.
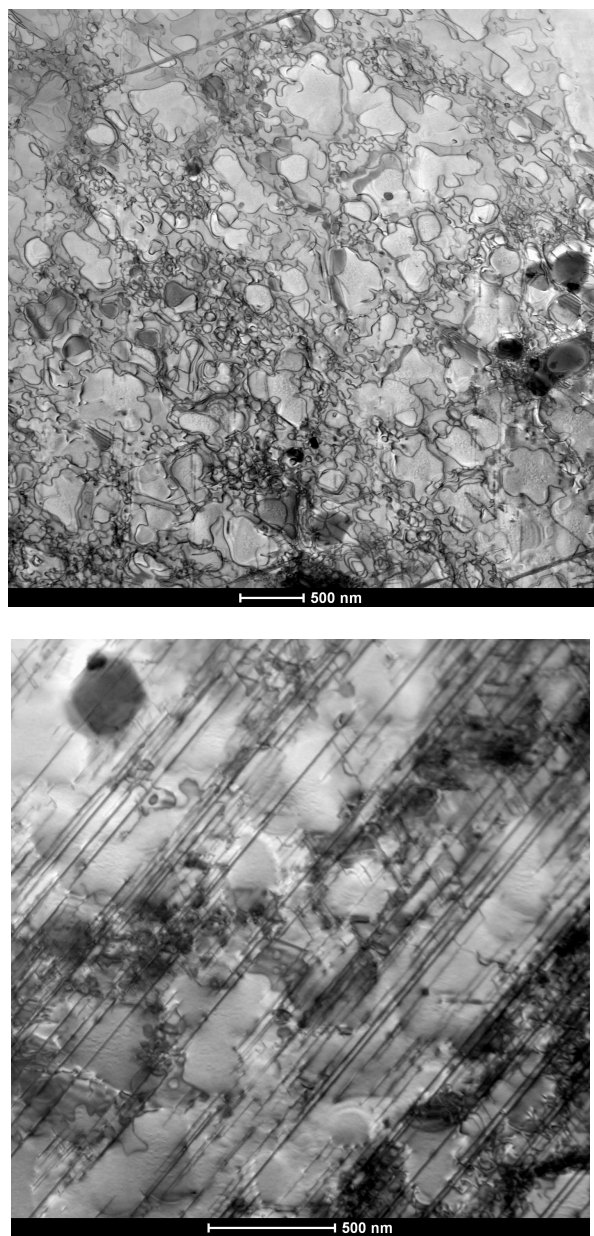

Figure 7. STEM imaging of dislocation activity in constant strain rate tests.

Another active mechanism in the constant strain rate tests is the presence of non-planar deformation (wavy slip), as shown in Figure 8. Wavy slip has not been previously identified during monotonic loading of polycrystalline Ni-based superalloys. It has, however, been observed following low cycle fatigue (LCF) of the same alloy (in terms of microstructure and composition) to that used in the present creep and constant strain rate tests [8]. Wavy slip was observed to be the dominant deformation mode in LCF tests performed at both $426{ }^{\circ} \mathrm{C}$ and $704{ }^{\circ} \mathrm{C}$. In these cases, wavy slip was found to be the result of repeated cross- and cubeslipping processes of paired dislocations, traversing between octahedral $\{111\}$ and cube $\{001\}$ planes, shearing the matrix and precipitates (Figure 8b).

The maximum stress reached during the LCF tests (> $896 \mathrm{MPa})$ is comparable to that achieved during the constant strain rate tests, and is considerably higher than the lower stress creep stresses; this suggests that there may be a minimum stress required to activate the wavy slip mechanism. Based on the previous LCF analysis, elevated temperature $\left(\geq 426^{\circ} \mathrm{C}\right)$ may also be necessary for wavy slip to occur.

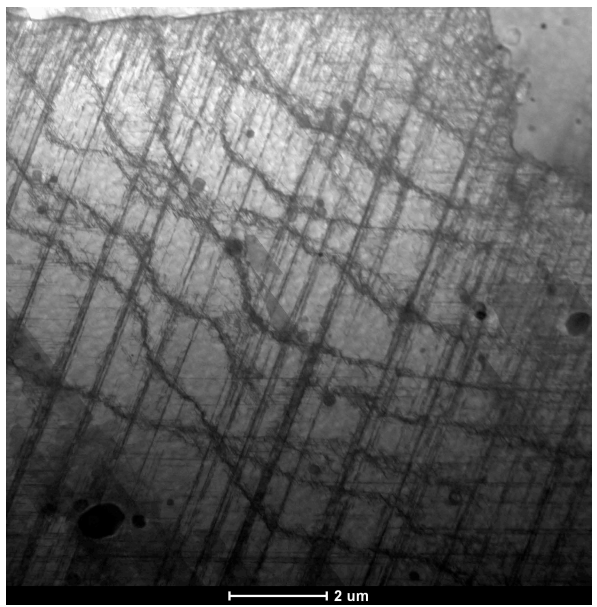

(a)

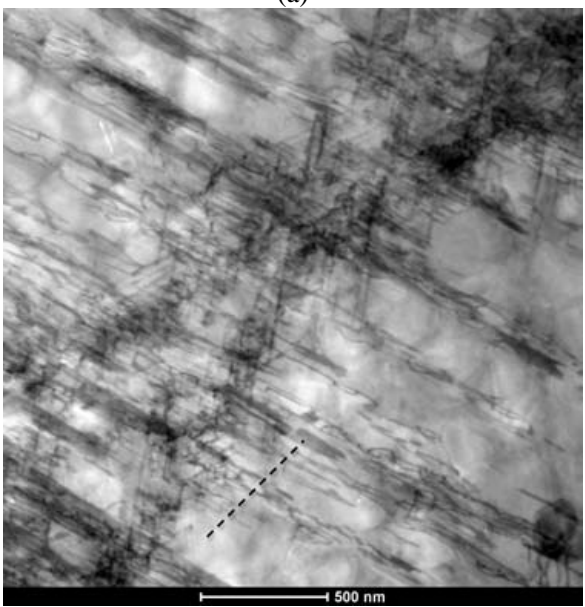

(b)

Figure 8. STEM imaging of a) wavy slip and b) cube slip in constant strain rate test (cube trace shown).

\section{Phase Field and Analytical Models of Dislocation Dissociation and De-Correlation}

Phase field simulations of dislocation-precipitate interactions were carried out in order to examine possible dislocation activities and deformation modes. These simulations were performed for a given $\gamma$-channel width and as a function of the orientation and magnitude of the in-plane shear stress. By incorporating the generalized stacking fault (GSF) energy of the octahedral slip plane [9], the phase field method describes naturally dislocation dissociation and formation of stacking faults in both ordered and disordered phases [10]. The GSF energy surfaces of the $\gamma$ and $\gamma$ ' phases are formulated using various fault energy data obtained from experiments and ab initio calculations, including intrinsic stacking fault (ISF), extrinsic stacking fault (ESF), anti-phase boundary (APB), complex stacking fault 
(CSF), superlattice intrinsic stacking fault (SISF) and superlattice extrinsic stacking fault (SESF) [11].

\section{Simulation Results}

The simulation results in Figure 9 show that a variety of deformation modes and associated dislocation configurations can be observed, including Orowan looping and APB shearing by a full dislocation, Orowan looping by both partial dislocations, Orowan looping by the leading partial only, and the arrest of both partials at the entrance of the $\gamma$ channel [6]. As indicated in the plot, each circle corresponds to one phase field simulation performed with the specified stress magnitude and orientation. It is clear that, depending on the orientation of the Burgers vector of the $a / 2\langle 110\rangle$ dislocation with respect to the applied stress, the leading Shockley partial can experience either a higher or lower resolved shear stress than that experienced by the trailing one.
In general, de-correlated motion of the partials may take place only if the leading partial has a higher resolved shear stress. Exceptions may exist when considering the reduction of the effective channel width for the trailing partial to pass through because of the dislocation segments in the channel deposited by the leading partial, especially for cases of either narrow channels or low ISF energy. Note that due to the extra gradient energy associated with the phase field description of dislocation under mesoscale, the dislocation line tension in the phase field simulations is benchmarked as the equation seen below [6]

$T=2.45 \frac{\mu b^{2}}{2}$

Thus, the stress level indicated in Figure 9 is relatively high in order to offset the high line tension.

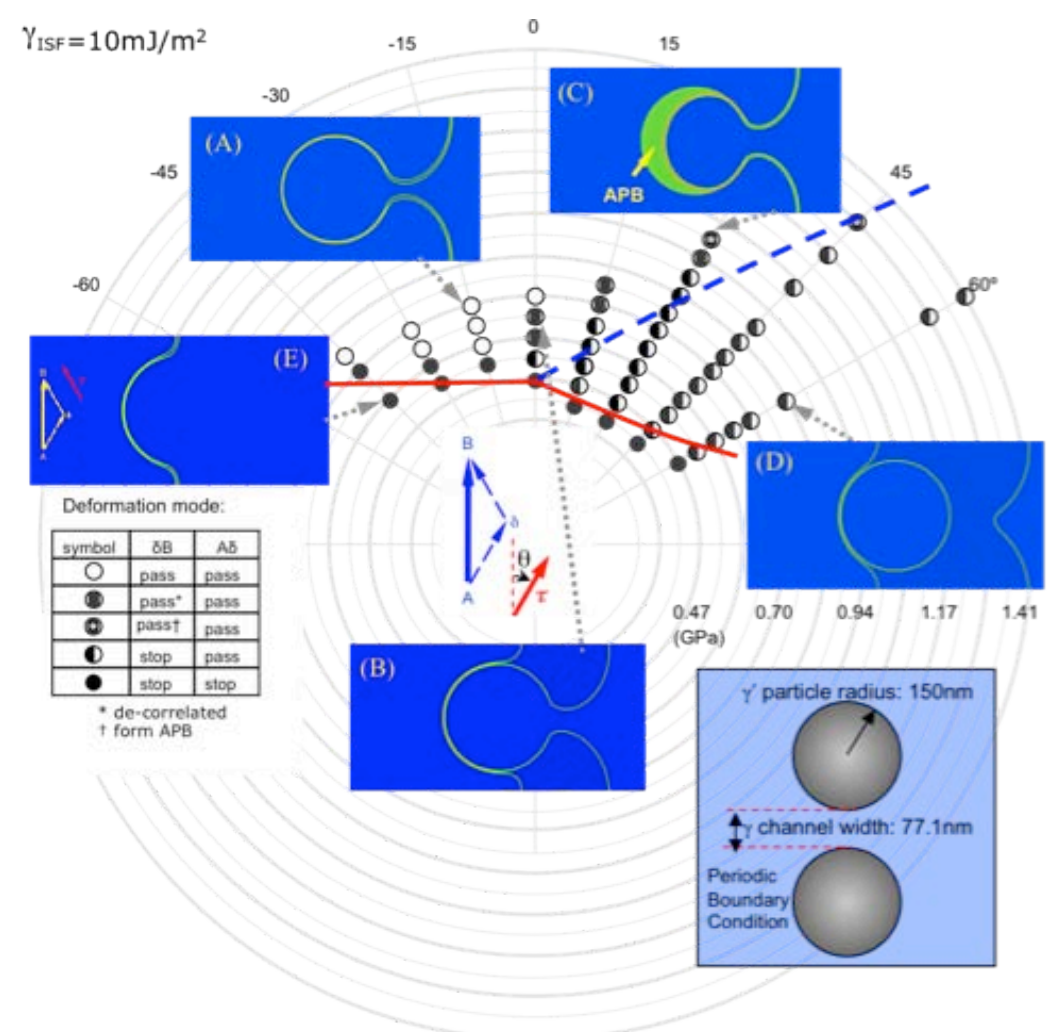

Figure 9: Phase field simulation of the interaction between a matrix dislocation $(a / 2<110>)$ and array of $\gamma$ ' particles. (A) Orowan bowing of full dislocation; (B) Orowan bowing of both partial dislocations (dislocation dissociation); (C) APB shearing of $\gamma^{\prime}$; (D) Orowan bowing of the leading partial dislocation (de-correlation); (E) dislocations arrested in front of the channel. The solid (red) curve is the critical stress for the leading dislocation to pass through the channel and the dashed (blue curve is the critical stress for the trailing dislocation to pass. For these simulations, the periodic boundary conditions create an array of spherical $\gamma$ ' particles with a radius of $150 \mathrm{~nm}$ and $\gamma$ channel width of $77.1 \mathrm{~nm}$. The shear modulus of the $\{111\}$ plane, $\mathrm{m}_{111}=67.2 \mathrm{GPa}$ for both the $\gamma$ and $\gamma$ ' phases with an intrinsic stacking fault (ISF) energy, $\mathrm{G}_{\mathrm{ISF}}=10 \mathrm{~mJ} / \mathrm{m}^{2}$. The Burgers vector for the perfect $a / 2<110>$ dislocation is along the vertical direction $\left(0^{\circ}\right)$, thus, the Burgers vector for the leading partial, $\mathrm{Ad}$, is along $+30^{\circ}$, and the trailing partial, $\mathrm{dB}$, is along $-30^{\circ}$ as illustrated. The angle, $\mathrm{q}$, between the in-plane shear stress and the Burgers vector of the perfect dislocation, $\mathrm{AB}$, can vary from $-180^{\circ}$ to $+180^{\circ}$. Note that for $\mathrm{q}>90^{\circ}$ or $\mathrm{q}<-90^{\circ}$, $\mathrm{dB}$ should be defined as the leading partial and the dislocation should be placed initially at the right side of the $\gamma^{\prime}$ particles. 
Inspired by the results obtained from phase field simulations, a reduced-order analytical model of dislocation dissociation in $\mathrm{Ni}$ based disk alloys was developed based on a line tension model of Orowan looping and elastic interactions between dislocations [6]. This analytical model not only can restore the dislocation line tension to experimental measured values, but also has high computation efficiency, thus enabling direct interface with crystal plasticity/finite element models (CP/FEM). Physical parameters such as shear modulus, $\gamma$-channel width, applied stress orientation and magnitude, intrinsic stacking fault energy $\left(\mathrm{G}_{\mathrm{ISF}}\right)$, and friction force created by solute drag or shearing of smaller tertiary particles can be easily incorporated in the calculation. As observed in the experiments [6], dissociated or de-correlated motion of partials eventually leads to microtwinning with Shockley partials passing on adjacent glide planes, shearing both $\gamma$ and $\gamma^{\prime}$ phase (short range re-ordering takes place in the $\gamma^{\prime}$ phase [12]).

Equipped with this dislocation dissociation (decorrelation) model, the average $\gamma$ channel width obtained from TEM/SEM experimental pictures can be combined with the specific loading condition in order to determine the favored deformation mode, i.e. full $a / 2\langle 110\rangle$ dislocation shearing or microtwinning associated with $a / 6<112\rangle$ partials. Parametric computation results indicate that increasing ISF energy or applied stress amplitude will reduce the probability of having dissociated (decorrelated) dislocation motion. Similarly, by making $\gamma$ channel wider one can achieve the same effect.

The influence of friction force caused by solute drag or cutting of small tertiary particles is convoluted with other parameters. Increasing the friction force raises the critical bypassing stress (as if applied stress is reduced), which promotes dislocation dissociation. However, as this change of bypassing critical stress is strongly orientation dependent, in some cases (especially for low applied stress with microstructures having relatively larger $\gamma$ channel width), higher friction force will actually inhibit dislocation dissociation, thus reducing the probability of deformation by microtwins in later stages. The parametric calculation results are summarized in Table I with respect to factors determining the dominant deformation mechanisms.

Table I. Factors Affecting Deformation Mechanisms

\begin{tabular}{|c|c|c|}
\hline \multirow{3}{*}{ ISF energy } & High & $\begin{array}{c}\text { a/2<110> } \\
\text { dislocation shearing }\end{array}$ \\
\cline { 2 - 3 } & Low & $\begin{array}{c}\text { ISF shearing of } \gamma \\
\text { and SESF shearing } \\
\text { of } \gamma^{\prime} \text { (microtwin) }\end{array}$ \\
\hline$\gamma$-channel width & Wide & $\begin{array}{c}\text { a/2<110> } \\
\text { dislocation } \\
\text { shearing }\end{array}$ \\
\cline { 2 - 3 } & Narrow & $\begin{array}{c}\text { ISF shearing of } \gamma \\
\text { and SESF shearing } \\
\text { of } \gamma^{\prime} \text { (microtwin) }\end{array}$ \\
\hline \multirow{3}{*}{ Load magnitude } & High & $\begin{array}{c}\text { a/2<110> } \\
\text { dislocation } \\
\text { shearing }\end{array}$ \\
\cline { 2 - 3 } & Low & $\begin{array}{c}\text { ISF shearing of } \gamma \\
\text { and SESF shearing } \\
\text { of } \gamma^{\prime} \text { (microtwin) }\end{array}$ \\
\hline
\end{tabular}

Two-Scale Homogenized Crystal Plasticity FEM Model for $\gamma$ $\gamma$ 'Microstructures

This section describes a multi-scale approach for developing grain scale crystal plasticity models from the homogenization of subgrain response of nickel based superalloy microstructural representative volume elements (RVE's) containing $\gamma-\gamma$ ' phases. Detailed information on microstructural morphology, in terms of the distribution of size, shape, spacing and volume fraction of the $\gamma^{\prime}$ phase, was incorporated in the grain level crystal plasticity model. The subgrain level constitutive model includes a dislocation density based crystal plasticity model for the $\gamma$ phase. In fact, the criteria for APB shearing of the $\gamma$ ' phase and flow rule for subsequent plastic deformation in the $\gamma$ ' phase after APB shearing are the essence of the new model.

In the lower scale, the geometrical features of the $\gamma-\gamma$ ' microstructure are explicitly considered in the dislocation density based crystal plasticity model. The development of statistically stored dislocations (SSD's) in the $\gamma$-channel due to various dislocation generation and annihilation mechanisms is considered. The evolution of geometrically necessary dislocations (GND's) at the locations with plastic inhomogeneity is considered through computation of the components of GND's from the plastic strain gradient. The mechanism of APB shearing of the $\gamma$ ' precipitates by the matrix dislocations are also captured through the new model.

The lower scale model was homogenized in order to be used in the next level (i.e., crystal level) of simulation. There is no explicit consideration of the $\gamma-\gamma$ ' microstructure at this level of simulation. Crystal plasticity parameters, such activation energy, passing stress and activation volume, are expressed as functions of microstructural variables like volume fraction, shape and channel width. At the grain level, the homogenized crystal plasticity based model along with a new model for micro-twin initiation and propagation has been developed. The parameters of the homogenized model are calibrated through comparison of simulation of model response with single crystal experimental data [13].

\section{Crystal Plasticity Results and Discussion}

The dislocation density based crystal plasticity model has been used to simulate the deformation behavior of individual grains of superalloys, as a precursor to more complete polycrystalline simulations. Due to the availability of single crystal creep experiments for the nickel based superalloy CMSX-4, contains approximately $70 \%$ volume fraction of cuboidal shaped $\gamma^{\prime}$ precipitates. The experiment was conducted at $800{ }^{\circ} \mathrm{C}$, with loading along the [001] direction. The results of simulation with and without activation of APB shearing of the precipitates are shown in comparison with experimental results at a strain rate of $10^{-3} \mathrm{~s}^{-1}$ in Figure 10(b). The response is very stiff if the mechanism of APB shearing is not activated in the constitutive model. With incorporation of the criteria for APB shearing and the corresponding constitutive relations for plastic flow in the $\gamma$ ' phase, the stress-strain response can be simulated satisfactorily. 


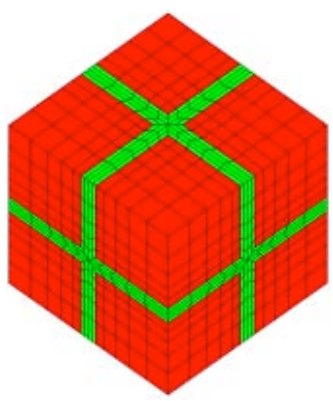

(a)

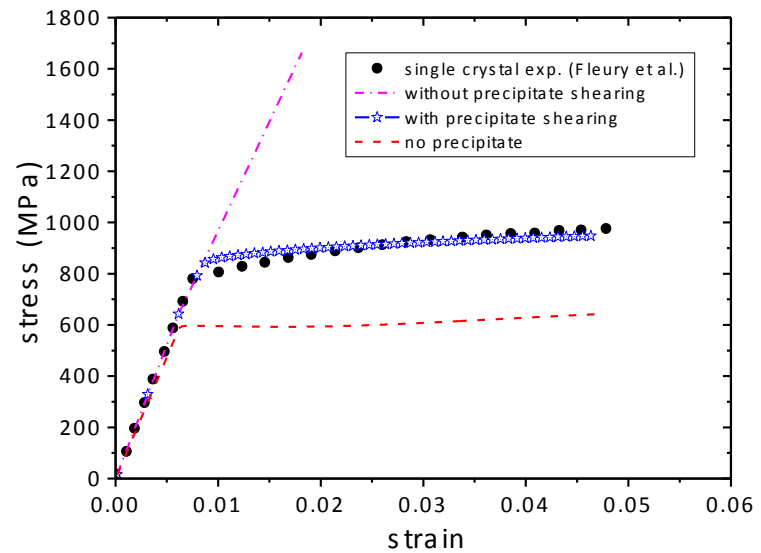

(b)

Figure 10. (a) RVE and finite element (FE) mesh of $\gamma-\gamma$ ' microstructure loaded along the vertical [001] direction (b) stressstrain response of a single crystal nickel based superalloy CMSX4 at $800^{\circ} \mathrm{C}$

The homogenized crystal plasticity model for single crystals, with the effect of precipitate shape and the volume fractions on yielding and plastic hardening response, was validated by experimental results of creep response of a single crystal nickel based superalloy in [14]. The test was conducted at $700^{\circ} \mathrm{C}$ and $820 \mathrm{MPa}$, which are similar testing parameters and therefore in a comparable deformation regime with the present experimental work. The alloy used contained $57.9 \%$ of cuboidal $(n=\infty) \gamma^{\prime}$ precipitates, and was loaded in [001] direction. The results of the simulation are compared with that of experiment, and are both plotted in Figure 11.

It can be observed that the dislocation density based crystal plasticity model is able to predict the experimental creep response very satisfactorily. The volume averaged slip strain rate in each slip system was evaluated, and its evolution (using the maximum of all the slip systems) with plastic work density is presented in Figure 12 for various values of precipitate volume fraction $\left(v_{p}\right)$ and shape factors $\left(\mathrm{n}_{1}\right)$. This data was used to derive the following homogenized parameters; homogenized activation energy, homogenized passing stress, and homogenized activation volume.

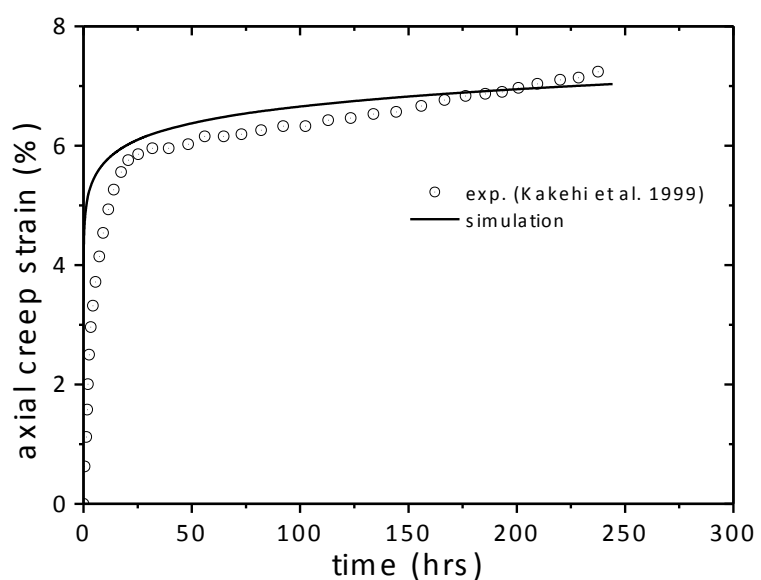

Figure 11. Simulation of creep response of CMSX-4 single crystal superalloy CMSX-4 at $700{ }^{\circ} \mathrm{C}$ and $820 \mathrm{MPa}$ of applied stress for tensile loading in [001] direction

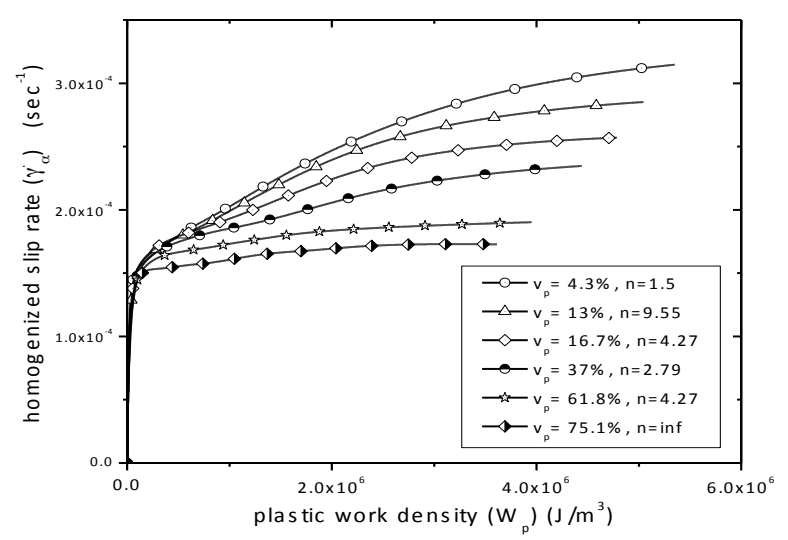

Figure 12. Variation of maximum homogenized slip rate with plastic work density as a function of volume fraction and shapes of precipitates

A novel, two-level crystal plasticity model for this class of superalloys is now being developed. This new model is motivated by the characterization and phase field modeling efforts, as well as the dislocation-based crystal plasticity model just described. A schematic illustrating the hierarchy in this multi-scale approach is shown in Figure 12, which is intended to simulate polycrystalline response while keeping the essential effects of microstructure and operative mechanisms. At the lower scale, a dislocation-densitybased crystal plasticity model simulates the response of various microstructures (shapes and volume fractions of $\gamma^{\prime}$ precipitates) within a single crystal, including a new model for simulation of the mechanism of APB shearing. The lower scale model is then homogenized as a function of various microstructural parameters and the homogenized model is used in the next level of crystal plasticity based simulation model. 


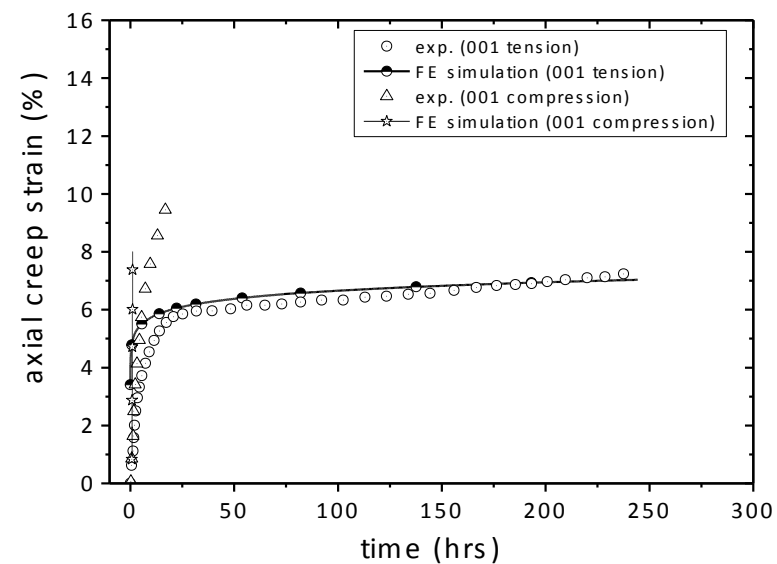

Figure 13. Prediction of tension-compression asymmetry in the creep response of the single crystal nickel based superalloy CMSX-4 due to loading in [001] direction in tension and compression.

At the second scale, a homogenized crystal plasticity model is then developed from the dislocation-density-based model, where hardness evolution laws are introduced to avoid explicit use of dislocation density representation in the constitutive laws. In addition, a new criterion for initiation of microtwinning based on the phase field results and a constitutive model for twin strain accumulation are developed. The model is also able to reproduce the tension-compression asymmetry as observed in single crystal experiments. This new modeling framework will be compared with both single crystal and polycrystalline response of ME3 under monotonic loading conditions.

Tension-compression asymmetry is successfully modeled with the formulation incorporating microtwinning. The creep response with time is predicted in Figure 13 and shows that the model is very close to those of experiment both in [001] tension and [001] compression modes.

\section{Discussion and Conclusions}

From the creep testing, it appears that there is a stress threshold at which non-planar activity appears. The lower stresses show a dominance of reorder-mediated deformation, especially microtwins. Diffuse dislocation bands also appear throughout the microstructure. At the higher stresses, non-planar wavy slip appears. This non-planar wavy slip provides a connection between the high stress creep tests, and the constant strain rate and LCF tests. Future work will also include exploring the effects of changing temperature and stress on the creep test strain rate, in order to measure important material parameters such as activation energy and stress exponent.

The strong crystal anisotropy effects that are predicted from the phase field modeling results motivate the need for single crystal testing in order to carefully map out material response and deformation mechanisms as a function of grain orientation. As a consequence, future work will also involve single grain specimens that are being harvested from large grained, directionally solidified polycrystalline ME3. This data will provide higher- fidelity information for the existing phase field and crystal plasticity modeling structures, bringing the model closer to the ultimate goal of modeling polycrystalline behavior.

Coupling the aforementioned modeling efforts to deformation characterization is necessary to gain further insight into the substructure development of advanced Ni-based disk superalloys. This link is particularly needed when the material exhibits a strong grain-to-grain (and thus, orientation) variation of operative mechanisms, as is the case for complex loading conditions discussed above.

\section{Acknowledgements}

The authors of this paper would like to acknowledge support from the Air Force Research Lab's Metals Affordability Initiative (MAI). GE Aviation and Pratt \& Whitney are also acknowledged for their support in the MAI program. The authors would also like to acknowledge the other universities on this project, Georgia Institute of Technology and the University of Rhode Island for their contributions.

\section{References}

1. P.L. Reynolds, et al., "Metals Affordability Initiative II Durable High Temperature Disk Material" (Report MAI Team/ Pratt \& Whitney, 2011).

2. E.J. Payton, P.J. Phillips, and M.J. Mills, "Semi-Automated Characterization of the $\gamma$ ' Phase in Nickel-based Superalloys via High-Resolution Backscattered Imaging," Materials Science and Engineering A, 527 (10-11) (2010), 2684-2692.

3. P.J. Phillips, M.J. Mills, and M. DeGraef, "Systematic Row and Zone Axis STEM Defect Image Simulations," Philosophical Magazine, 91 (16) (2011), 2081-2101.

4. R. R. Unocic, et al., "Deformation Mechanisms in Ni-Base Disk Superalloys at Higher Temperatures," Superalloys 2008. Proceedings of the International Symposium on Superalloys (Champion, PA, 2008), 377-385.

5. L. Kovarik, et al., "The Intermediate Temperature Deformation of Ni-Base Superalloys: Importance of Reordering," JOM, 61 (2) (2009), 42-48.

6. R. R. Unocic, et al., "Dislocation Decorrelation and Relationship to Deformation Microtwinning During Creep of a $\gamma$ ' Precipitate Strengthened Ni-Based Superalloy," Acta Materialia, 59 (19) (2011), 7325-7339.

7. G. B. Viswanathan, et al., "Investigation of Creep Deformation Mechanisms at Intermediate Temperatures in Rene 88 DT," Acta Materialia, 53 (2005), 3041-3057.

8. P.J. Phillips, et al., "Low Cycle Fatigue of a Ni-Based Superalloy: Planar Deformation," Scripta Materialia, 62 (10) (2010), 790-793.

9. V. Vitek, "Intrinsic Stacking Faults in Body-Centered Cubic Crystals," Philosophical Magazine, 154 (18) (1968), 773-786. 
10. C. Shen and Y. Wang, "Incorporation of $\gamma$ Surface to Phase Field Model of Dislocations: Simulating Dislocation Dissociation in FCC Crystals," Acta Materialia, 52 (3) (2004), 683-691.

11. N. Zhou, et al., "Modeling Displacive-Diffusional Coupled Dislocation Shearing of $\gamma$ ' Precipitates in Ni-Based Superalloys," Acta Materialia, 59 (9) (2011), 3484-3497.

12. M. Kolbe, "The High Temperature Decrease of the Critical Resolved Shear Stress in Nickel-Based Superalloys," Materials Science and Engineering A, 319-321 (2001), 383-387.

13. A. Ma, F. Roters, D. Raabe, "A Dislocation Density Based Constitutive Model for Crystal Plasticity FEM Including Geometrically Necessary Dislocations," Acta Materialia, 54 (8) (2006), 2169-2179.

14. N. Tsuno, et al., "Tension/Compression Asymmetry in Yield and Creep Strengths of Ni-Based Superalloys," Superalloys 2008. Proceedings of the International Symposium on Superalloys (Champion, PA, 2008), 433-442. 